\title{
Decomposition of Graphs into Paths
}

\author{
Fábio Botler ${ }^{1}$ \\ Orientadora: Yoshiko Wakabayashi ${ }^{2}$
}

${ }^{1}$ Departamento de Ingeniería Industrial - FCFM-UChile Avenida Republica, 701 - CEP 8370439 - Santiago - Chile

${ }^{2}$ Departamento de Ciência da Computação - IME-USP

Rua do Matão, 1010 - CEP 05508-090 - São Paulo - Brazil

\{fbotler,yw\}@ime.usp.br

\begin{abstract}
We study the Decomposition Conjecture posed by Barát and Thomassen (2006), which states that, for each tree $T$, there exists a natural number $k_{T}$ such that, if $G$ is a $k_{T}$-edge-connected graph and $|E(T)|$ divides $|E(G)|$, then $G$ admits a partition of its edge set into copies of $T$. In a series of papers, Thomassen has verified this conjecture for stars, some bistars, paths of length 3 , and paths whose length is a power of 2. In this paper we prove this conjecture for paths of any given length. Our technique is then used to prove weakenings of a conjecture of Kouider and Lonc (1999), and a conjecture of Favaron, Genest and Kouider (2010), both for path decomposition of regular graphs.
\end{abstract}

\section{Introduction}

A set $\mathcal{D}=\left\{H_{1}, \ldots, H_{k}\right\}$ of pairwise edge-disjoint subgraphs of a graph $G$ is called a decomposition of $G$ if these subgraphs cover the edge set of $G$. If $H_{i}$, for $1 \leq i \leq k$, is isomorphic to a fixed graph $H$, then we say that $\mathcal{D}$ is an $H$-decomposition of $G$. When $H=P_{2}$ is a path with two edges, one can prove that a connected graph $G$ admits an $H$-decomposition if and only if $G$ has an even number of edges. On the other hand, Dor and Tarsi (1997) proved that deciding whether a graph admits an $H$-decomposition is an NP-complete problem whenever $H$ has a component with at least 3 edges. It is then natural to look for sufficient conditions for a graph to admit an $H$-decomposition. As we will see, this problem has attracted the attention of many researchers. The focus of this paper is the study of the $H$-decomposition problem when $H=P_{\ell}$ is a path of length $\ell$. To tackle this problem, we developed a technique that consists in finding first a decomposition of the given graph into trails (some of which may be paths), and then, by means of a Disentangling Lemma, switching the edges between the elements of the current decomposition, so that the new decomposition contains more paths than the original one. This technique of finding first a trail decomposition, and then using this lemma, has shown to be useful to attack other path decomposition problems. In fact, we [Botler et al. 2015c] first used this idea to decompose triangle-free 5-regular graphs into paths of length 5. Later, we were able to explore better this technique to obtain path decomposition of two important family of graphs, namely, regular graphs and highly edge-connected graphs. Our proofs use a generalization of the technique we presented in [Botler et al. 2016], which combines a method introduced by Thomassen [Thomassen 2008a] and a technique used by Lovász [Lovász 1968] for decomposition into cycles and paths. 
In Section 2, we study path decompositions of regular graphs, and present results related to the Conjectures of Kouider and Lonc (1999), and Favaron, Genest, and Kouider (2010). In Section 3, we study path decompositions of highly edge-connected graphs, mentioning our main result: the proof of the Decomposition Conjecture of Barát and Thomassen (2006) for paths.

Owing to space limitation, we only mention the main results and ideas contained in [Botler 2016]. For more details on the results of Section 2, the reader is referred to [Botler et al. 2015b]; full proofs of the results in Section 3 are given in [Botler et al. 2017a].

The basic terminology and notation used in this paper are standard (see, e.g. [Diestel 2010]). A path $P$ in $G$ is a sequence of distinct vertices $P=v_{0} v_{1} \cdots v_{\ell}$ such that $v_{i} v_{i+1} \in E(G)$, for $i=0,1, \ldots, \ell-1$. The length of $P$ is the number of its edges. A path of length $\ell$ is denote by $P_{\ell}$. A vanilla trail is a trail $v_{0} v_{1} \cdots v_{\ell}$ such that $v_{1} \cdots v_{\ell-1}$ is a path. A vanilla $\ell$-trail is a vanilla trail of length $\ell$.

\section{Decomposition of regular graphs into paths of fixed length}

In 1964, Ringel conjectured that the complete graph $K_{2 \ell+1}$ admits a $T$-decomposition for any tree $T$ with $\ell$ edges. This conjecture is commonly confused with the Graceful Tree Conjecture that says that every tree $T$ with $n$ vertices admits a labeling $f: V(T) \rightarrow$ $\{0, \ldots, n-1\}$ such that $\{1, \ldots, n-1\} \subseteq\{|f(x)-f(y)|: x y \in E(T)\}$. In fact, the latter implies Ringel's Conjecture (see [Rosa 1967]), and this fact implies that Ringel's Conjecture holds for many classes of trees, such as stars, paths, bistars, caterpillars, and lobsters (see [Edwards and Howard 2006]). Häggkvist (1989) generalized Ringel's Conjecture for regular graphs as follows.

Conjecture 2.1 (Graham-Häggkvist, 1989). For each tree $T$ with $\ell$ edges, if $G$ is a $2 \ell$ regular graph, then $G$ admits a T-decomposition.

In 1989, Häggkvist also proved that Conjecture 2.1 holds when the girth of $G$ is at least the diameter of $T$. In the case of paths, Kouider and Lonc (1999) improved Häggkvist's result, proving that a $2 \ell$-regular graph with girth $g \geq(\ell+3) / 2$ admits a $P_{\ell}$-decomposition $\mathcal{D}$ such that every vertex is the end-vertex of exactly two paths of $\mathcal{D}$. They also conjectured that this statement holds for every $2 \ell$-regular graph.

Conjecture 2.2 (Kouider-Lonc, 1999). Every 2l-regular graph admits a $P_{\ell^{-}}$ decomposition $\mathcal{D}$ such that each vertex is the end-vertex of exactly two paths of $\mathcal{D}$.

We say that a path decomposition $\mathcal{D}$ of a graph is balanced if there is a positive integer $k$ such that each vertex is the end-vertex of exactly $k$ paths of $\mathcal{D}$. Heinrich, Liu and $\mathrm{Yu}$ (1999) proved that if $G$ is a $3 m$-regular graph that contains an $m$-factor, then $G$ admits a balanced $P_{3}$-decomposition. In [Botler and Talon 2017] it is proved that Conjecture 2.2 holds for $\ell=4$ (see Theorem 2.4). In [Botler et al. 2017b] we prove a weakening of Conjecture 2.2, which states that, for each positive integers $\ell$ and $g$ such that $g \geq 3$, there is an $m_{0}=m_{0}(\ell, g)$ such that, if $G$ is a $2 m \ell$-regular graph with $m \geq m_{0}$ and girth at least $g$, then $G$ admits a balanced $P_{\ell}$-decomposition. The next theorem gives a bound for $m_{0}$.

Theorem 2.3. Let $\ell, g$ and $m$ be positive integers such that $g \geq 3$ and let $G$ be a $2 m \ell$ regular graph with girth at least $g$. If $m>\lfloor(\ell-2) /(g-2)\rfloor$, then $G$ admits a balanced $P_{\ell^{-}}$decomposition. 
Theorem 2.4. Every 8-regular graph admits a balanced $P_{4}$-decomposition.

Another result related to those stated above is due to Kotzig (1957), who proved that a 3-regular graph $G$ admits a $P_{3}$-decomposition if and only if $G$ contains a perfect matching. Favaron, Genest, and Kouider (2010) proved that if $G$ is a 5-regular graphs without cycles of length 4 and containing a perfect matching, then $G$ admits a $P_{5}$-decomposition. They also conjectured that Kotzig's result may be generalized in the following way.

Conjecture 2.5 (Favaron-Genest-Kouider, 2010). For every odd positive integer $\ell$, if $G$ is an $\ell$-regular graph that contains a perfect matching, then $G$ admits a $P_{\ell}$-decomposition.

In this case, the degree of the vertices of the graph is decreased by one-half, but a perfect matching is required. In [Botler et al. 2015c], we extended Favaron, Genest, and Kouider's result, proving that triangle-free 5-regular graphs that contain a perfect matching admit a $P_{5}$-decomposition. A natural generalization of perfect matching is the concept of $m$-factor. An $m$-factor of a graph $G$ is an $m$-regular spanning subgraph of $G$. In [Botler et al. 2017b], we also prove the following result, which is a weakening of Conjecture 2.5: for each positive integers $\ell$ and $g$ such that $\ell$ is odd and $g \geq 3$, there is an $m_{0}=m_{0}(\ell, g)$ such that, if $G$ is an $m \ell$-regular graph with $m \geq m_{0}$, girth at least $g$, and containing an $m$-factor, then $G$ admits a balanced $P_{\ell^{-}}$-decomposition. We also give a bound for $m_{0}$. This value is stated in the next theorem.

Theorem 2.6. Let $\ell, g$ and $m$ be positive integers such that $\ell$ is odd and $g \geq 3$, and let $G$ be an $m \ell$-regular graph with girth at least $g$ that contains an $m$-factor. If $m>$ $2\lfloor(\ell-2) /(g-2)\rfloor$, then $G$ admits a balanced $P_{\ell^{-}}$decomposition.

It would be interesting to prove, if possible, a better bound for $m_{0}$. We showed that when $g=\ell-1$ the bound on $m_{0}$ can be improved to 1 , which proves Conjecture 2.5 for graphs with sufficiently high girth, and generalizes the result in [Botler et al. 2015c].

Theorem 2.7. For every odd positive integer $\ell$, if $G$ is an $\ell$-regular graph with girth at least $\ell-1$ and containing a perfect matching, then $G$ admits a $P_{\ell}$-decomposition.

In what follows, we mention the main ideas used in the proof of Theorem 2.3.

Sketch of the proof of Theorem 2.3. Let $\ell, g, m$ and $G$ be as in the statement. The proof follows by induction on $\ell$. The statement holds trivially for $\ell=1$, and the proof for the case $\ell=2$ follows simply by choosing an Eulerian orientation of $G$ and decomposing the out-going edges at each vertex into paths of length 2 . Thus, we can suppose that $\ell \geq 3$. Using a theorem of Petersen (1891), we can show that $G$ contains a $4 m$-factor $H$. Thus, $G^{\prime}=G-E(H)$ is a $2 m(\ell-2)$-regular graph with girth at least $g$, and $m>\lfloor((\ell-2)-$ $2) /(g-2)\rfloor$. By the induction hypothesis, $G^{\prime}$ admits a balanced $P_{\ell-2}$-decomposition $\mathcal{D}^{\prime}$. It is easy to see that since $\mathcal{D}^{\prime}$ is balanced, each vertex of $G$ is the end-vertex of precisely $2 m$ paths of $\mathcal{D}^{\prime}$. Moreover, if we choose an Eulerian orientation for $H$, then we have $d_{H}^{+}(v)=$ $d_{H}^{-}(v)=2 m$ for every vertex $v$ of $G$. Thus, using the edges of $H$ we can extend each path of $\mathcal{D}^{\prime}$ with one out-going edge at each of its end-vertices, obtaining a decomposition $\mathcal{D}$ of $G$ into vanilla $\ell$-trails. The Disentangling Lemma in [Botler 2016] is then used to transform $\mathcal{D}$ into a balanced $P_{\ell}$-decomposition of $G$, concluding the proof.

\section{Decomposition of highly edge-connected graphs into paths of fixed length}

In this section we study $H$-decomposition of highly edge-connected graphs. When $H$ is a tree, Barát and Thomassen (2006) conjectured that high edge-connectivity (together with 
the obvious necessary condition on the number of edges) may be sufficient for a graph to admit an $H$-decomposition.

Conjecture 3.1. For any fixed tree $T$, there exists a natural number $k_{T}$ such that, if $G$ is a $k_{T}$-edge-connected graph and $|E(G)|$ is divisible by $|E(T)|$, then $G$ admits a $T$ decomposition.

Barát and Thomassen (2006) proved that Conjecture 3.1 in the special case $T$ is the claw $K_{1,3}$ is equivalent to a weakening of Tutte's 3-flow conjecture, posed by Jaeger (1988). Recently, Lovász, Thomassen, Wu, and Zhang (2013) proved that a $(3 k-3)$-edge-connected graph $G$ admits a $K_{1, k}$-decomposition if $|E(G)|$ is divisible by $k$, showing that Conjecture 3.1 holds for stars, and, in particular, confirming Jaeger's weak 3-flow conjecture. Between 2008 and 2013, Thomassen also proved that Conjecture 3.1 holds for paths of length 3 , paths of length 4 , a family of bistars, and more recently, for paths whose length is a power of 2. Recently, Barát and Gerbner (2014) and Thomassen (2013a) proved that it is sufficient to prove Conjecture 3.1 for bipartite graphs. That is, Conjecture 3.1 is equivalent to the following conjecture.

Conjecture 3.2. For any fixed tree $T$, there exists a natural number $k_{T}^{\prime}$ such that, if $G$ is a bipartite $k_{T}^{\prime}$-edge-connected graph and $|E(G)|$ is divisible by $|E(T)|$, then $G$ admits a T-decomposition.

In [Botler et al. 2016], we proved that Conjecture 3.1 holds for paths of length 5 . This result was also obtained by Merker [Merker 2017], who, additionally, verified Conjecture 3.1 for trees with diameter at most 4. Finally, in [Botler et al. 2017a], we proved Conjecture 3.1 for paths of any given length. For that, we first proved Conjecture 3.2 for paths of any length, and then used the equivalence of Conjectures 3.1 and 3.2.

Theorem 3.3. Let $\ell$ be a positive integer, and let $r=\max \{32(\ell-1), \ell(\ell+1)\}$. If $G$ is a $2(13 \ell+4 r-4)$-edge-connected bipartite graph such that $|E(G)|$ is divisible by $\ell$, then $G$ admits a $P_{\ell}$-decomposition.

Theorem 3.4. Let $\ell$ be a positive integer, $r=\max \{32(\ell-1), \ell(\ell+1)\}$, and put $k_{T}^{\prime}=$ $2(13 \ell+4 r-4)$. If $G$ is a $\left(4 k_{T}^{\prime}+16 \ell^{6 \ell+1}\right)$-edge-connected graph such that $|E(G)|$ is divisible by $\ell$, then $G$ admits a $P_{\ell}$-decomposition.

The proof of Theorem 3.3 follows the structure of the proof of Theorem 2.3. For that, we define new concepts such as fractional factors and $\mathbb{F}$-balanced decompositions, which extend the concepts of factors and balanced decompositions, respectively, to the scope of highly edge-connected graphs.

\section{Concluding remarks}

Graph decomposition is a topic that has shown to be rich in conjectures and challenging problems that have brought significant contributions to structural graph theory. In this work we developed a technique to deal with decompositions of graphs into paths that has shown to be useful to deal with well-studied problems (Conjectures 2.1, 2.5, and 3.1). Furthermore, the tools developed in this work have led us to other new results as in [Botler and Talon 2017].

When we were writing the main result of Section 3 in [Botler et al. 2017a], we learned that Bensmail, Harutyunyan, Le, and Thomassé (2015) obtained a similar result using a different approach. Recently, together with Merker, these authors [Bensmail et al. 2017] proved Conjecture 3.1 using probabilistic tools. This shows 
that the study of graph decompositions may be explored with different approaches, each of which contributes to enrich the area of structural graph theory.

We plan to continue working on Conjecture 2.1. We also would like to generalize the Disentangling Lemma to deal with more general structures, seeking for results analogous to the ones in Section 2 for other structures. In another direction, we believe that it is possible to improve the girth condition of Conjecture 2.5.

We conclude mentioning that the results obtained in [Botler 2016] have been published in the Journal of Combinatorial Theory, Series B [Botler et al. 2017a] and Discrete Mathematics [Botler et al. 2015c]; and have been accepted to the European Journal of Combinatorics [Botler et al. 2017b] and Discrete Applied Mathematics [Botler et al. 2016], the first one being one the most prestigious journals in combinatorics. We have also presented these results in many international conferences, among which we mention ICGT 2014, LAGOS 2015, EuroComb 2015 [Botler et al. 2015b, Botler et al. 2015a].

\section{References}

Barát, J. and Gerbner, D. (2014). Edge-decompositions of graphs into copies of a tree with four edges. Electron. J. Combin., 21(1):Research Paper 55 pp. (electronic).

Barát, J. and Thomassen, C. (2006). Claw-decompositions and Tutte-orientations. J. Graph Theory, 52(2):135-146.

Bensmail, J., Harutyunyan, A., Le, T., Merker, M., and Thomassé, S. (2017). A proof of the Barát-Thomassen conjecture. J. Comb. Theory, Ser. B, 124:39-55.

Bensmail, J., Harutyunyan, A., Le, T.-N., and Thomassé, S. (2015). Edge-partitioning a graph into paths: beyond the Barát-Thomassen conjecture. ArXiv e-prints.

Botler, F. (2016). Decomposição de Grafos em Caminhos. PhD thesis, Instituto de Matemática e Estatística - Universidade de São Paulo.

Botler, F., Mota, G., Oshiro, M., and Wakabayashi, Y. (2015a). Decompositions of highly connected graphs into paths of any given length. Electronic Notes in Discrete Mathematics, 49:795 - 802 .

Botler, F., Mota, G., Oshiro, M., and Wakabayashi, Y. (2015b). Path decompositions of regular graphs with prescribed girth. Electronic Notes in Discrete Mathematics, 49:629 $-636$.

Botler, F., Mota, G. O., Oshiro, M. T. I., and Wakabayashi, Y. (2016). Decomposing highly connected graphs into paths of length five. Discrete Appl. Math., to appear.

Botler, F., Mota, G. O., Oshiro, M. T. I., and Wakabayashi, Y. (2017a). Decomposing highly edge-connected graphs into paths of any given length. J. Combin. Theory Ser. $B, 122: 508-542$.

Botler, F., Mota, G. O., Oshiro, M. T. I., and Wakabayashi, Y. (2017b). Decomposing regular graphs with prescribed girth into paths. European Journal of Combinatorics, to appear.

Botler, F., Mota, G. O., and Wakabayashi, Y. (2015c). Decompositions of triangle-free 5-regular graphs into paths of length five. Discrete Math., 338(11):1845-1855. 
Botler, F. and Talon, A. (2017). Decomposing 8-regular graphs into paths of length 4. Discrete Mathematics, 340(9):2275 - 2285.

Diestel, R. (2010). Graph theory, volume 173 of Graduate Texts in Mathematics. Springer, Heidelberg, fourth edition.

Dor, D. and Tarsi, M. (1997). Graph decomposition is NP-complete: a complete proof of Holyer's conjecture. SIAM J. Comput., 26(4):1166-1187.

Edwards, M. and Howard, L. (2006). A survey of graceful trees. Atl. Electron. J. Math., 1(1):5-30.

Favaron, O., Genest, F., and Kouider, M. (2010). Regular path decompositions of odd regular graphs. J. Graph Theory, 63(2):114-128.

Häggkvist, R. (1989). Decompositions of complete bipartite graphs. In Surveys in combinatorics, 1989 (Norwich, 1989), volume 141 of London Math. Soc. Lecture Note Ser., pages 115-147. Cambridge Univ. Press, Cambridge.

Heinrich, K., Liu, J., and Yu, M. (1999). $P_{4}$-decompositions of regular graphs. J. Graph Theory, 31(2):135-143.

Jaeger, F. (1988). Nowhere-zero flow problems. In Selected topics in graph theory, 3, pages 71-95. Academic Press, San Diego, CA.

Kotzig, A. (1957). Aus der Theorie der endlichen regulären Graphen dritten und vierten Grades. Casopis Pěst. Mat., 82:76-92.

Kouider, M. and Lonc, Z. (1999). Path decompositions and perfect path double covers. Australas. J. Combin., 19:261-274.

Lovász, L. (1968). On covering of graphs. In Theory of Graphs (Proc. Colloq., Tihany, 1966), pages 231-236. Academic Press, New York.

Lovász, L. M., Thomassen, C., Wu, Y., and Zhang, C.-Q. (2013). Nowhere-zero 3-flows and modulo $k$-orientations. J. Combin. Theory Ser. B, 103(5):587-598.

Merker, M. (2017). Decomposing highly edge-connected graphs into homomorphic copies of a fixed tree. J. Comb. Theory, Ser. B, 122:91-108.

Petersen, J. (1891). Die Theorie der regulären graphs. Acta Math., 15(1):193-220.

Ringel, G. (1964). Problem n.25. In Theory of Graphs and its Applications (Proc. Sympos. Smolenice, 1963). Publ. House Czechoslovak Acad. Sci., Prague.

Rosa, A. (1967). On certain valuations of the vertices of a graph. In Theory of Graphs (Internat. Sympos., Rome, 1966), pages 349-355. Gordon and Breach, New York.

Thomassen, C. (2008a). Decompositions of highly connected graphs into paths of length 3. J. Graph Theory, 58(4):286-292.

Thomassen, C. (2008b). Edge-decompositions of highly connected graphs into paths. Abh. Math. Semin. Univ. Hambg., 78(1):17-26.

Thomassen, C. (2013a). Decomposing a graph into bistars. J. Combin. Theory Ser. B, 103(4):504-508.

Thomassen, C. (2013b). Decomposing graphs into paths of fixed length. Combinatorica, 33(1):97-123. 